Journal of Patient-Centered

Volume 4

Issue 4 - Health Disparities and Inequities: Part

Article 16 I

$11-6-2017$

\title{
Identifying and Targeting Age-Related Colorectal Cancer Screening Rate Disparities in Family Medicine Residency Clinics
}

\author{
Jonathan J. Blaza \\ Jasmine R. Wiley \\ Matthew Gill \\ Alonzo Jalan \\ Will Lehmann \\ Deborah Simpson \\ Jeffrey A. Stearns
}

Follow this and additional works at: https://aah.org/jpcrr

Part of the Community Health and Preventive Medicine Commons, Family Medicine Commons, Health Services Research Commons, Medical Education Commons, and the Oncology Commons

\section{Recommended Citation}

Blaza JJ, Wiley JR, Gill M, Jalan A, Lehmann, W, Simpson D, Stearns JA. Identifying and targeting agerelated colorectal cancer screening rate disparities in family medicine residency clinics. J Patient Cent Res Rev. 2017;4:258-9.

Published quarterly by Midwest-based health system Advocate Aurora Health and indexed in PubMed Central, the Journal of Patient-Centered Research and Reviews (JPCRR) is an open access, peer-reviewed medical journal focused on disseminating scholarly works devoted to improving patient-centered care practices, health outcomes, and the patient experience. 
receiving the alert, ED pharmacists reviewed the patient profile, including differential diagnosis; antibiotic allergies, reactions and/ or previous tolerance; recent bacterial cultures; and any antibiotics already ordered, to assess dose and spectrum of coverage. Pharmacists intervened as needed to ensure patients received appropriate broad spectrum antibiotics. Antibiotics were defined as broad spectrum in the same manner as by the Centers of Medicare and Medicaid Services in its Early Management Bundle, Severe Sepsis/Septic Shock. Education was provided to physicians, nurses and pharmacists to encourage timely administration. Outcomes to be measured include mean time to broad spectrum antibiotics, percentage of patients who received broad spectrum antibiotics within 1 hour of presentation, and percentage of patients who received broad spectrum antibiotics within 3 hours of presentation. Results: Pre-alert intervention, patients who were coded with the ICD-10 codes for sepsis, severe sepsis or septic shock in May $2016(n=65)$ were analyzed to determine mean time to broad spectrum antibiotics $(2.81 \pm 1.63$ hours $)$, percentage of patients who received broad spectrum antibiotics within 1 hour $(3.1 \%)$, and percentage of patients who received broad spectrum antibiotics within 3 hours $(67.7 \%)$. Post-alert outcomes will be reported at Aurora Scientific Day.

Conclusion: To be reported at Aurora Scientific Day.

\section{Mechanisms of Left Ventricular Thrombus Formation in Heart Failure With Reduced Ejection Fraction: Novel Insights From Two-Dimensional Speckle Tracking Echocardiography}

Dhruv Chawla, Tracy Hammonds, Tadele Mengesha, Matt Umland, Khawaja Afzal Ammar, Vinay Thohan

\section{Aurora Cardiovascular Services; Aurora Research Institute}

Background: Patients suffering from heart failure with reduced ejection fraction (HFrEF) are at increased risk for left ventricular (LV) thrombus and subsequent thromboembolism, yet anticoagulation is not routinely recommended for left ventricular ejection fraction (LVEF) alone. We sought to determine the role of two-dimensional speckle tracking echocardiography (2D-STE) to quantify regional changes in cardiac function associated with LV thrombus, which may prospectively guide anticoagulation.

Purpose: Help enable cardiovascular clinicians to use 2D-STE to evaluate regional strain patterns among patients with HFrEF with and without LV thrombus. Our results suggest that statistically lower regional longitudinal strain patterns in a well-matched cohort identified patients with thrombus. The postulate that these patterns existed prior to the formation of thrombus remains to be tested. Our results warrant further investigation with a larger prospective cohort. Methods: We retrospectively identified patients with $\mathrm{LVEF} \leq 35 \%$ who had LV thrombus $(\mathrm{n}=12)$ and a matched (in demographics and LVEF) cohort who did not have LV thrombus $(n=36)$. We performed offline 2D-STE longitudinal strain analysis. Descriptive statistics were used to compare variables.

Results: The average age of identified patients was $62.7 \pm 15.0$ years; $71 \%$ were male. LVEF was not statistically different. LV enddiastolic diameter was increased in LV thrombus group (6.2 \pm 1.1 $\mathrm{cm}$ vs $5.5 \pm 0.65 \mathrm{~cm}, \mathrm{P}=0.014)$. $\mathrm{LV}$ thrombus group had consistently reduced regional strain in the inferior wall $(-5.3 \pm 3.9$ vs $-8.3 \pm 4.1$, $\mathrm{P}=0.033)$, at the apex $(-5.3 \pm 4.4$ vs $-8.9 \pm 4.0, \mathrm{P}=0.012)$, and in the left anterior descending coronary distribution $(-5.3 \pm 3.4$ vs $-7.8 \pm 3.4, \mathrm{P}=0.031)$. There was no significant difference in global longitudinal strain or strain dispersion.
Conclusion: Among patients with HFrEF, LV thrombus is associated with reduced longitudinal strain in inferior and apical regions and in the left anterior descending territory.

\section{Challenges in Delivering Refugee Health Systems}

Thy Vo, Fabiana Kotovicz

University of Wisconsin School of Medicine and Public Health; Department of Family Medicine, Aurora University of Wisconsin Medical Group

Background: Aurora Health Care is the major health care system providing care to refugees in Milwaukee, where half of Wisconsin's refugee population resides. Like many other institutions caring for refugee patients, Aurora faces significant challenges when trying to address refugee health needs. Even with the assistance of medical interpreters, cultural differences, language barriers and limited patient health literacy, as well as lack of knowledge of refugee patients' backgrounds, are major obstacles encountered by health care providers in this setting.

Purpose: This quality improvement study aims to assess Aurora providers' perceptions of the benefits and barriers to working with refugee patient populations.

Methods: An online survey was distributed to health care providers and staff at two academic family practice clinics before and after a 5-session educational series. Each educational session delivered monthly focused on refugee cultural awareness. Mental health providers and former refugees working as case managers or interpreters provided education about the main refugee populations in Wisconsin and the refugee resettlement process. The survey assessed participants' perceptions about providing health care to refugees. Participants were asked to respond to questions on a Likert scale from 1 to 7 (ie, strongly disagree to strongly agree) and to two questions comprised of rank choices investigating barriers to effective health care delivery. Responses to questions on Likert scale $\geq 5$ were lumped into an agreement category, while all others were considered a disagreement. Fisher's exact test was used to compare pre- and posteducation responses.

Results: Perception about new medical knowledge and cultural competency had statistically significant increase comparing pre- and postintervention data $(\mathrm{P}=0.0474)$. Insufficient interpreter services and insufficient time for appointments were ranked the top barriers to providing health care services to refugees before and after intervention. Participants also ranked refugees' poor understanding of the U.S. health care system as the biggest challenge in delivering care for refugee patients before and after intervention, followed by communication with resettlement agencies in the preintervention survey and cultural differences in the postintervention survey.

Conclusion: Promotion of cultural awareness and proficiency within groups delivering primary care to refugees can be a valuable tool when trying to overcome obstacles.

Identifying and Targeting Age-Related Colorectal Cancer Screening Rate Disparities in Family Medicine Residency Clinics

Jonathan J. Blaza, Jasmine R. Wiley, Matthew Gill, Alonzo Jalan, Will Lehmann, Deborah Simpson, Jeffrey A. Stearns

Departments of Family Medicine and Academic Affairs, Aurora University of Wisconsin Medical Group 
Background: Health care systems continuously seek to improve patient care through population-level analysis of clinical quality metrics and patient characteristics to identify disparities in care. Nationally, disparities in colorectal cancer (CRC) screening rates have been identified with lower screening rates reported for patients who are uninsured and/or lower socioeconomic status, African American/ black, Asian, and non-English-speaking Hispanic patients. No agerelated CRC screening rate disparities with associated interventions have been reported.

Purpose: Determine and address CRC screening disparities in care provided to eligible patients $>50$ years old in two primary care residency clinics.

Methods: Retrospective analysis using REAL-G (race, ethnicity, age, preferred language, gender) categories and insurance coverage was completed on a 12-month data set to identify presence of CRC screening disparities. Barriers to CRC screening for largest disparity gap were then identified by clinic staff at two family medicine residency clinics (a third primary care clinic in same zip code and service region were used for nonintervention comparison) using the Institute for Healthcare Improvement fishbone approach. The project team, informed by the literature, then identified and implemented targeted interventions, monitoring progress during a 6-month period. Interventions included provider education with periodic reminders regarding system-approved CRC screening options and a workflowbased intervention. Postintervention analysis was completed using same preintervention approach.

Results: The largest CRC screening disparity for region and clinics was associated with age, with screening gaps ranging from $13 \%$ to $15 \%$ between populations aged $50-54$ years versus $>65$ years. CRC screening rate disparities by race, ethnicity, and gender were less than $10 \%$. Postintervention, one targeted clinic had a $6 \%$ increase in the CRC screening rates in the target population (age: 50-54) while a second targeted clinic had a $1 \%$ increase in screening rates during this period. The comparison primary care residency clinic had a $1 \%$ decline in CRC screening rates. Differences in insurance utilization types for CRC screening rates by clinic were noted. Differences between targeted clinic screening rates were attributed to successful workflow implementation and provider/staff champions.

Conclusion: Analyzing population data at a micro/clinic level using REAL-G categories can inform targeted interventions that aim to reduce health disparity gaps.

\section{Variations in Practice of Apnea Test for Brain Death: Review From a Multihospital Health Care System}

\section{Jeffrey Stein, Marvi V. Rijhwani, Ernesto Brauer \\ Department of Internal Medicine, Aurora Sinai Medical Center; Bellin Health Pulmonary Clinic, Bellin Health Systems}

Background: Ventilation encompasses both active and passive processes. Air is initially drawn into the lungs due to a negative intrathoracic pressure created using the respiratory muscles, most importantly the diaphragm. In contrast, expiration is the passive relaxation of the respiratory muscles. Oxygenation occurs when oxygen diffuses across the alveolar-capillary membrane. The ability to oxygenate without ventilation has been termed apneic diffusion oxygenation or apneic oxygenation. We believe it is crucial to keep alveoli open in order for adequate oxygenation to occur. This can be achieved with the aid of positive end-expiratory pressure (PEEP). We investigated this concept in patients who are brain-dead because they cannot ventilate. The stimulus to breathe originates from chemoreceptors in the brainstem. These cells respond to a decrease in $\mathrm{pH}$ by triggering the body to take a breath. A positive apnea test confirms that the patient has no functioning brainstem.

Purpose: Determine the rate of $\mathrm{pO}_{2}$ and $\mathrm{pCO}_{2}$ changes during different methods of the apnea test and identify variations in practice within Aurora Health Care.

Methods: Data were collected retrospectively on brain-dead patients older than 18 years. Data points pulled from Epic medical records included serial arterial blood gases (ABGs) that were completed during the apnea test and patient demographics. The rate of change in $\mathrm{pCO}_{2}$ and $\mathrm{pO}_{2}$ was evaluated using both Mann-Whitney and two-sample t-tests comparing a PEEP valve group to all other oxygenation methods.

Results: Eight variations of the test were performed, with median starting $\mathrm{CO}_{2}$ for the oxygenation and PEEP group of 43 and 44 $\mathrm{mmHg}$, respectively ( $95 \%$ confidence interval: $26-53, \mathrm{P}=0.6771)$. Oxygenation group had a mean $\mathrm{CO}_{2}$ increase of $2.95 \mathrm{mmHg} /$ minute, whereas the PEEP valve group increased at $4.60 \mathrm{mmHg} /$ minute. No statistical significance was found $(\mathrm{P}=0.0508)$. Neither was there significant difference between the rate of desaturation between the oxygenation and PEEP valve group $(6.53 \mathrm{mmHg}$ vs $2.60 \mathrm{mmHg}$, respectively; $\mathrm{P}=0.5536$ ).

Conclusion: We found no difference in the rate of $\mathrm{CO}_{2}$ increase comparing the oxygenation group to the PEEP valve group. This suggests that there is no significant component of $\mathrm{CO}_{2}$ washout in the lungs using the PEEP valve setup. A superior method of apneic oxygen was not able to be demonstrated with our results due to an insufficient sample size and practice variations. The most common method to perform the apnea test at our institutions is preoxygenation. Effects of Revision Surgery on Grade of Adverse Local
Tissue Reaction Following Recall of a Modular Hip Implant

\section{Joseph F. Davies, Danielle M. Greer, Susan Truchan}

Aurora Advanced Orthopedics; Center for Urban Population Health; Aurora University of Wisconsin Medical Group; Aurora Research Institute

Background: The Stryker Rejuvenate modular hip implant device allows for greater versatility in matching a patient's anatomy than conventional implants. Device recalls and in vivo metal hypersensitivity after total hip arthroplasty (THA) are common. However, recall of the Rejuvenate implant represents one of the largest recall volumes to date, highlighting our uncertainty regarding causes of device metal fretting/corrosion and adverse local tissue reaction (ALTR). While devices with metal-on-metal bearings historically were culprits for release of metal debris, more recently developed modular-neck devices add opportunity for adjacent metal components to rub together. With the Rejuvenate device, corrosion or fretting likely occurs at the cobalt-chromium neck and titanium stem interface. Cobalt $(\mathrm{Co})$ and chromium $(\mathrm{Cr})$ particles then irritate tissues locally and cause a gradation of problems or indications of ALTR.

Purpose: Quantify the effect of revision surgery on ALTR grade in patients who previously underwent THA receiving the recalled Rejuvenate hip implant.

Methods: We conducted a prospective observational study of all patients who underwent THA performed by a single Aurora Health Care orthopedic surgeon using the Rejuvenate implant. Following implant recall in July 2012, patients were notified via letter/phone and asked to visit regularly (every 3-6 months) for lab work, imaging and device assessment. Using repeated measures multinomial logit analysis we examined the effect of revision surgery on abnormal grade of ALTR (ie, grade 1-4 vs 0), adjusting 